\title{
L'apport des modèles animaux en biologie cellulaire
}

$>$ Si la culture cellulaire a été et reste le matériel préféré du biologiste cellulaire - essentiellement Yves Bobinnec de par sa facilité d'utilisation - les cellules mises en culture perdent certaines de leurs propriétés : et en acquièrent d'autres. De plus, ces mêmes cellules ne représentent qu'une partie d'un tissu et $a$ fortiori d'un organe, et les résultats obtenus dans des conditions de culture ne peuvent pas être extrapolés à des conditions plus complexes. $\varepsilon$ n conséquence, le chercheur a dû faire appel à des modèles animaux pour résoudre les problèmes qui lui étaient posés, la question devenant celle du choix du modèle. Les principaux systèmes existants sont décrits dans ce texte de façon non exhaustive, sous forme d'exemples, en illustrant leurs apports en biologie cellulaire. <

Le matériel privilégié du biologiste cellulaire est la cellule en culture, facile à manipuler et à entretenir en laboratoire. Les cultures cellulaires ont permis d'importantes avancées dans la compréhension de l'architecture d'une cellule animale, des mécanismes de division cellulaire, du transport intracellulaire et de l'organisation des différents organites cellulaires. Toutefois, ces cellules, le plus souvent immortalisées, ont adopté un comportement particulier qui s'accompagne, entre autres, de la perte d'un certain nombre de propriétés telles que le contrôle strict du cycle cellulaire et de la répartition des chromosomes ou la mise en place des communications intercellulaires. Certains résultats obtenus sur ces lignées peuvent ainsi ne pas être retrouvés dans le contexte bien plus complexe d'un tissu. II devient alors nécessaire de faire appel à un modèle animal, plus sophistiqué, dont le choix dépend de l'objectif.

L'histoire de la recherche en biologie cellulaire est émaillée de faits marquants obtenus grâce au développement de modèles animaux. Ce texte entend dresser un rapide panorama des principaux systèmes existants. Pour chacun d'eux, un exemple est choisi pour illustrer l'apport de ce

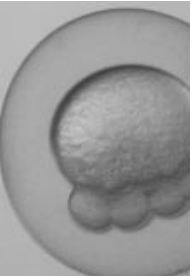

modèle dans cette discipline scientifique.

L'émergence d'un nouveau modèle animal provient d'abord de la volonté d'un expérimentateur de résoudre un problème pour lequel cet animal semble particulièrement adapté. La survie du modèle dépendra de sa capacité effective à se prêter à l'analyse biologique, puisque la création d'une communauté scientifique d'un « certain poids » travaillant sur ce modèle lui permettra de devenir une référence. À l'origine, le modèle animal doit répondre à un premier critère essentiel: être adapté, à moindre frais, à l'élevage en laboratoire. En fonction des objectifs scientifiques, un certain nombre de critères secondaires vont apparaître. Un exemple du choix que doit faire le biologiste face à un changement de modèle est parfaitement décrit par François Jacob lorsqu'il se trouva confronté à ce problème en 1967: «Comment choisir parmi les organismes favoris des embryologistes: oursin, grenouille, mouche, souris...? Chacun d'entre eux se prêtait à un type particulier d'expérimentation, mais pas ou peu à d'autres. Je pris un jour une feuille de papier pour noter toutes les propriétés qu'il me semblait souhaitable de trouver chez un animal pour répondre au type de recherche que je voulais engager: facilité d'élevage, vitesse de reproduction, simplicité d'analyse génétique, culture de cellules, études physiologiques développées, biochimie facile, possibilité d'étudier le comportement... II était clair que l'animal n'existait pas. Pour répondre à ces exigences, il eût fallu un hybride de grenouille, oursin, mouche...! » .

Aujourd'hui, la majeure partie de la communauté scientifique travaille avec une vingtaine de modèles animaux de 
référence. La souris, le poulet, le xénope et le poisson zèbre représentent les quatre grands modèles pour l'étude des vertébrés. La mouche drosophile et le ver Caenorhabditis elegans sont les modèles d'invertébrés les plus utilisés. La levure, l'algue Chlamydomonas ou la paramécie constituent les modèles d'unicellulaires les plus travaillés. Parmi les animaux marins, on peut citer l'oursin, l'étoile de mer, la palourde ou bien encore l'ascidie. La comparaison de la taille de quelques jeunes embryons appartenant à ces systèmes expérimentaux est présentée sur la Figure 1.

La souris constitue un modèle de choix pour l'étude des maladies humaines, en particulier celles d'origine génétique. Ce n'est pas le mammifère le plus proche de l'homme, mais l'un des plus petits et dont le cycle de reproduction est parmi les plus rapides ( 9 semaines). Son élevage est donc relativement aisé, même si une « animalerie souris » constitue une structure encore souvent complexe et coûteuse. Le développement embryonnaire est interne, comme pour tous les mammifères, ce qui ne facilite pas son analyse. La souris se prête cependant à l'analyse génétique, en particulier à travers l'inactivation sélective de certains gènes. Cette technique permet d'étudier les conséquences, au niveau d'un organisme, de la suppression d'une protéine ayant une fonction cellulaire. Un exemple récent concerne l'étude de la protéine $\mathrm{p} 53$. Le gène correspondant a été défini par des études menées sur des cellules en culture comme un gène suppresseur de tumeurs, dont l'inactivation entraîne des défauts de contrôle du cycle cellulaire et l'apparition de cancers. La protéine p53 est l'un des nombreux acteurs cellulaires impliqués dans la vérification du déroulement correct de la ségrégation du matériel génétique pendant le cycle cellulaire. Les souris, privées de ce gène à la suite de modifications génétiques, développent très tôt des tumeurs. À l'inverse, les animaux chez lesquels la protéine est produite en trop grande quantité se montrent plus résistants à l'apparition de cancers. Cependant, ils présentent également des signes de vieillissement prématuré, comme si les mécanismes cellulaires de lutte contre la cancérisation étaient en partie responsables du vieillissement. Cette observation n'aurait bien sûr pas pu être obtenue par la seule étude de ces mécanismes dans des lignées cellulaires en culture: l'analyse menée au niveau d'un organisme vivant apporte donc une information supplémentaire inestimable.

Le xénope est une grenouille d'origine africaine facile à élever en laboratoire. Cette espèce a été utilisée dans les années 1940 dans des tests de grossesse. L'urine d'une femme était injectée à une grenouille femelle; si la femme était enceinte, les hormones de grossesse contenues dans son urine déclenchaient la ponte chez le xénope. Pour le biologiste cellulaire, les œufs de xénope sont intéressants car particulièrement volumineux et faciles à injecter, mais
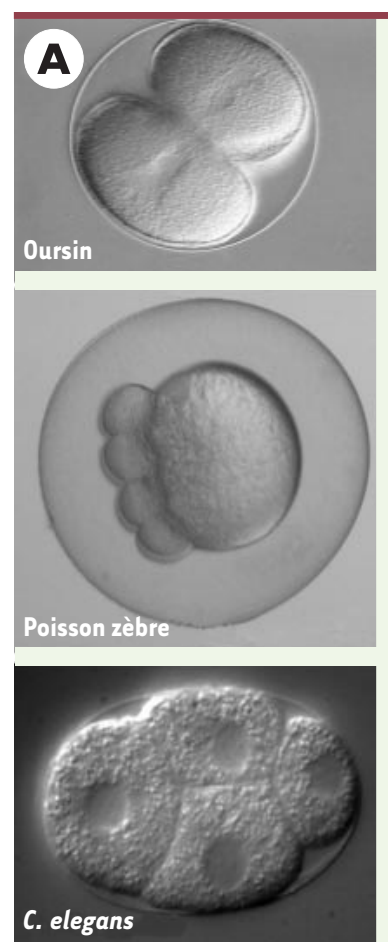
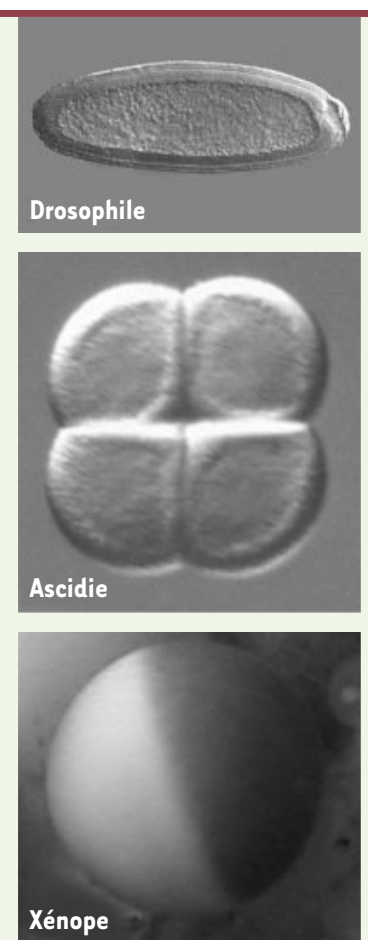

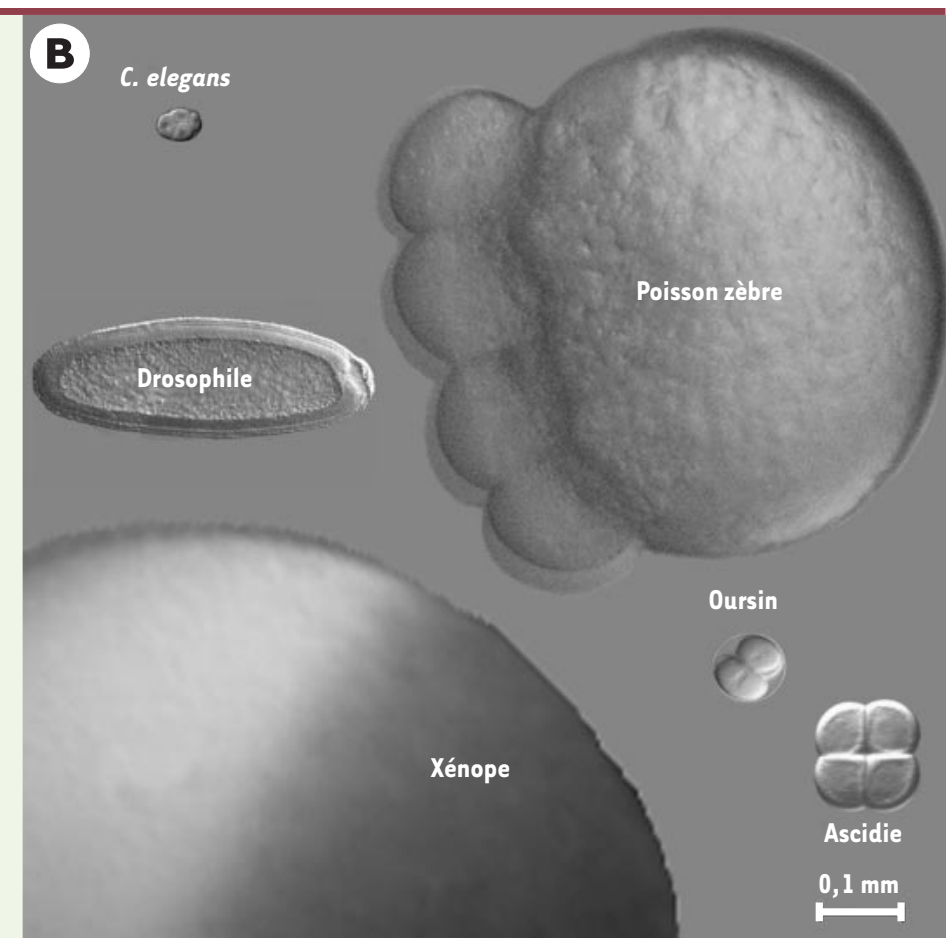

Figure 1. Quelques exemples d'organismes de référence, pendant les premiers stades embryonnaires. A. Oursin au stade 2 cellules; poisson zèbre au stade 8 cellules; C. elegans au stade 4 cellules; drosophile au stade «cellularisé » (6000 cellules); ascidie au stade 4 cellules; œuf de xénope non fécondé. $B$. Ces mêmes embryons sont présentés avec leur taille relative les uns par rapport aux autres. L'ensemble est grossi 90 fois. 
opaques au microscope, ce qui représente un handicap pour l'étude du développement des premières cellules embryonnaires. De plus, si le développement embryonnaire à partir de la fécondation est rapide, la maturation des œufs chez la femelle est extrêmement longue (10 à 12 mois), empêchant tout recours à des manipulations génétiques. Cela étant, une seule femelle pond une grande quantité d'œufs, dont on peut faire démarrer le développement artificiellement, ce qui permet de récolter une population parfaitement synchrone pour un stade particulier du développement embryonnaire. Ce modèle est donc idéal pour des expériences de biochimie qui nécessitent une quantité importante de matériel biologique. C'est grâce à cette abondante source de protéines qu'ont été caractérisés certains des acteurs de la régulation du cycle cellulaire (maturation promoting factor et cyclines) par Tim Hunt, prix Nobel de Médecine en 2001.

La drosophile est une mouche de 2 à 3 millimètres de long dont le cycle de développement est très rapide (environ quinze jours). Sa reproduction a été adaptée en laboratoire au tout début du XXe siècle. Thomas Hunt Morgan l'adopta comme système expérimental en 1909 pour clarifier les bases de la transmission de différents caractères d'une génération à l'autre. À cette époque, il était déjà pressenti que les chromosomes étaient responsables de l'hérédité, mais les facteurs en cause étaient totalement inconnus. Thomas Hunt Morgan et ses collaborateurs, regroupés dans leur laboratoire surnommé « la pièce des mouches », ont commencé à collecter des individus dont les traits morphologiques différaient de ceux des souches d'origine. Le premier mutant de drosophile ainsi isolé fut une mouche aux yeux blancs, et non rouges comme chez la souche sauvage, mutation très utilisée de nos jours comme marqueur génétique. Rapidement, Morgan et ses collaborateurs avanceront la théorie du « gène », unité responsable d'un trait donné, et découvriront que ces gènes se regroupent en quatre classes définies selon les propriétés de liaisons des uns aux autres, classes représentant les quatre chromosomes de la mouche. Toute la génétique moderne a été fondée sur la base de ces travaux, et ce n'est pas un hasard si la drosophile fut l'un des premiers organismes dont le génome a été entièrement séquencé; il est aujourd'hui possible de visualiser directement au niveau de l'ADN l'ensemble de la carte génétique définie par Thomas Hunt Morgan. La drosophile est un système expérimental particulièrement intéressant pour les biologistes. Pour le généticien, ce système bénéficie de la possibilité de manipuler des segments de gènes par utilisation d'éléments génétiques mobiles, les transposons, servant aussi bien à créer des mutations qu'à détecter des gènes ou des activateurs de gènes. Pour le biologiste cellulaire, la gigantesque collection de mutants disponibles, ainsi que la possibilité d'identifier les facteurs recherchés par comparaison avec la séquence du génome sont des atouts précieux. L'un des « défauts » de la drosophile est néanmoins la difficulté d'obtenir des quantités suffisantes d'extraits protéiques pour des analyses biochimiques conséquentes.

Deux types de levure se partagent le rôle de modèle de référence pour les unicellulaires, dont la force réside dans leur grande capacité à se prêter aux manipulations génétiques. Le plus utilisé est la levure de boulanger, Saccharomyces cerevisiae, dont le génome a été intégralement séquencé il y a plus de cinq ans. Le projet, aujourd'hui achevé, de réaliser l'inactivation systématique de chaque gène s'est rapidement mis en place. Ce système expérimental simple a contribué de façon notable à une meilleure compréhension de nombreux aspects de la biologie cellulaire et moléculaire, notamment par l'approche génétique mise en œuvre par Leland Hartwell (prix Nobel de Médecine 2001) dès les années 1970 et 1980, qui a permis de mieux comprendre la régulation du cycle cellulaire. Paul Nurse, troisième lauréat du prix Nobel de Médecine 2001, utilise quant à lui Schizosaccharomyces pombe pour décrypter ces mêmes mécanismes de contrôle du cycle cellulaire. Cette levure présente la caractéristique de se diviser par fission médiane, à la différence de $S$. cerevisiae qui se divise par bourgeonnement. Par cet aspect, et par d'autres comme la structure de son cycle cellulaire, la grande taille de ses chromosomes et leur condensation au cours de la mitose, S. pombe présente plus d'homologies fonctionnelles avec les eucaryotes supérieurs que $S$. cerevisiae et peut, pour certaines applications, représenter un meilleur modèle d'étude.

Le nématode Caenorhabditis elegans représente un système expérimental récent, introduit en 1965 par Sydney Brenner (prix Nobel de Médecine 2002) pour étudier le fonctionnement du système nerveux au sein d'un organisme simple. C. elegans est un petit ver rond, de un millimètre de long, qui se nourrit de bactéries et peut être, de façon relativement simple, cultivé sur une couche de gel en boîte de Pétri. Par ailleurs, les œufs et les tissus de C. elegans sont transparents, ce qui permet l'identification et le suivi des cellules au cours du développement. Il existe deux sexes, mâle et hermaphrodite. Au cours de leur développement, les hermaphrodites commencent par produire du sperme, qui est stocké dans une spermathèque, puis des ovocytes qui seront fécondés au contact de la spermathèque. Les mâles produisent uniquement du sperme et peuvent féconder les hermaphrodites. Grâce à une propriété encore non élucidée, le sperme provenant du mâle est dominant sur le sperme de l'hermaphrodite. Le cycle de vie de l'animal est de trois jours à température ambiante. Un hermaphrodite pond jusqu'à 300 œufs fécondés. L'un 
des objectifs du projet de Sydney Brenner était de décrire précisément le devenir de chaque cellule, de l'embryon jusqu'à la différenciation terminale. Par chance, le lignage cellulaire de cette espèce de nématode s'est révélé invariant, ce qui n'est pas le cas de ses cousins: ainsi, telle cellule du muscle ou du système nerveux provient toujours de la même cellule d'origine. À la fin de son développement, l'hermaphrodite possède exactement 959 cellules somatiques. Une autre facette du projet consistait en la description systématique, par microscopie électronique, de l'architecture des 302 neurones et des 56 cellules associées composant le système nerveux. Ces dernières années, la génétique de $C$. elegans s'est considérablement développée, fournissant nombre d'outils pour manipuler les six chromosomes de cet organisme. Récemment, un consortium international a achevé le séquençage de son génome. Cette masse considérable d'informations collectées peut avantageusement être utilisée grâce à la technique d'« interférence ARN », découverte justement chez C. elegans. Cette méthode fait appel à l'effet inhibiteur de l'injection d'un ARN double brin (ARN messager et son image) sur la traduction de la protéine correspondante. Cette technique d'interférence ARN a plus tard été adaptée à la drosophile et aux cellules de mammifères en culture.

Plus que dans toute autre discipline biologique, les avan- cées en biologie cellulaire dépendent du développement des techniques: l'émergence de nouveaux modèles d'étude, en particulier animaux, a déjà permis, et permettra encore des progrès remarquables dans la connaissance du fonctionnement de la cellule. $\nabla$

\section{SUMMARY}

Role of animal models in cell biology

Mainly because of their easy use, cell culture has been and remains a useful material for cellular biologist. Nevertheless, these cells loose or gain specific properties when they are cultured in vitro. Moreover, they represent only part of tissue and, a fortiori, of organs, so that results obtained in cell culture cannot strictly reflect that exist in more complex conditions. As a consequence, researcher has to use animal models, the question being now the choice of the animal model. In this paper, the main existing models are described and examples were given to illustrate their contribution to cell biology. $\diamond$

\section{RÉFÉRENCES}

1. Jacob F. La souris, la mouche et l'homme. Paris: Éditions Odile Jacob, 1997.

2. Tyner SD, Venkatachalam S, Choi J, et al. p53 mutant mice that display early ageing-associated phenotypes. Nature 2002; 415: 45-53.

3. Deutsch J. La drosophile: des chromosomes aux molécules. Montrouge: John Libbey Eurotext, 1994.

4. Fire A, Xu S, Montgomery MK, Kostas SA, Driver SE, Mello CC. Potent and specific genetic interference by doublestranded RNA in Caenorhabditis elegans. Nature 1998; 391: 744-5.
TIRÉS À PART

Y. Bobinnec

\section{Bon de commande}

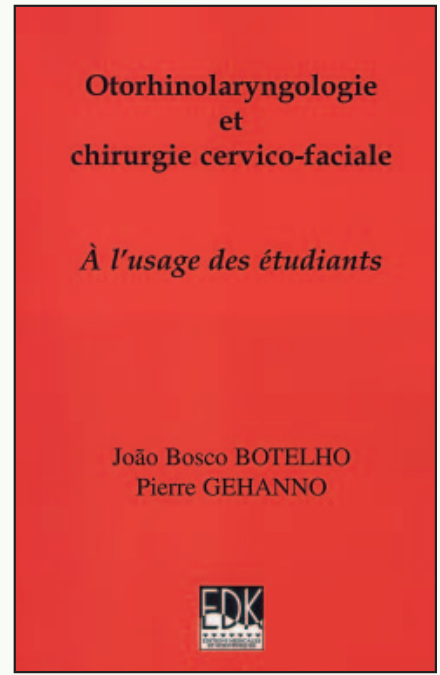

ISBN : 2-84254-075-1 160 pages
À retourner à EDK, 10 Villa d'Orléans - 75014 PARIS

Tél.: 0153910606 - Fax: 0153910607 - E-mail : editorial@edk.fr

NOM:

Prénom:

Adresse :

Code postal:

Ville:

Pays:

Fonction:

Je souhaite recevoir l'ouvrage ORL et chirurgie cervico-faciale: $30 €+3 €$ de port $=\mathbf{3 3} €$ TTC

en exemplaire, soit un total de $€$

$\square$ Par chèque, à l'ordre de $\mathbf{E} \mathbf{D} \mathbf{K}$

Par carte bancaire: $\square$ Visa $\square$ Eurocard/Mastercard $\square$ American Express

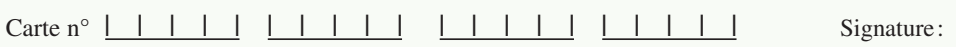

Date d'expiration: 